\title{
OPERATORS AND THE SPACE OF INTEGRABLE SCALAR FUNCTIONS WITH RESPECT TO A FRÉCHET-VALUED MEASURE
}

\author{
ANTONIO FERNÁNDEZ and FRANCISCO NARANJO
}

(Received 21 May 1997; revised 10 October 1997)

Communicated by P. G. Dodds

\begin{abstract}
We consider the space $L^{1}(v, X)$ of all real functions that are integrable with respect to a measure $\nu$ with values in a real Fréchet space $X$. We study L-weak compactness in this space. We consider the problem of the relationship between the existence of copies of $\ell^{\infty}$ in the space of all linear continuous operators from a complete DF-space $Y$ to a Fréchet lattice $E$ with the Lebesgue property and the coincidence of this space with some ideal of compact operators. We give sufficient conditions on the measure $v$ and the space $X$ that imply that $L^{1}(v, X)$ has the Dunford-Pettis property. Applications of these results to Fréchet AL-spaces and Köthe sequence spaces are also given.
\end{abstract}

1991 Mathematics subject classification (Amer. Math. Soc.): primary 46A04, 46A40, 46G10, 47B07.

Keywords and phrases: Fréchet lattice, L-weak compactness, Dunford-Pettis property, L-weakly compact operators, generalized AL-spaces, Köthe sequences spaces.

\section{Introduction}

In this paper we study operators with values in, or defined on, spaces of scalar-valued integrable functions with respect to a vector measure with values in a real Fréchet space.

This kind of integration was introduced by Lewis in [19] and developed essentially by Kluvánek and Knowles in [18] for locally convex spaces. Let us recall briefly the basic definitions (see [19] and [18]).

Throughout the paper $X$ will be a real Fréchet space. Denote by $\mathscr{W}_{0}(X)$ the system of all 0 -neighborhoods in $X$. Given $U \in \mathscr{U}_{0}(X)$ we denote by $p_{U}$ the associated

This research has been partially supported by La Consejería de Educación y Ciencia de la Junta de Andalucía and the DGICYT project no. PB94-1460.

(C) 1998 Australian Mathematical Society $0263-6115 / 98 \$ A 2.00+0.00$ 
Minkowski functional, that is,

$$
p_{U}(x)=\sup \left\{\left|\left\langle x^{\prime}, x\right\rangle\right|, x^{\prime} \in U^{\circ}\right\}, \quad x \in X,
$$

where $U^{\circ}$ denotes the polar set of $U$. Consider a countably additive measure $\nu$ : $\Sigma \rightarrow X$ defined on a $\sigma$-algebra $\Sigma$ of subsets of a non-empty set $\Omega$. For every 0 neighborhood $U$ in $X$ the $U$-semivariation of $v$ is the set function $\|v\|_{U}: \Sigma \rightarrow[0, \infty)$ defined by

$$
\|v\|_{U}(A):=\sup \left\{\left|x^{\prime} \nu\right|(A), x^{\prime} \in U^{\circ}\right\}
$$

where $\left|x^{\prime} \nu\right|$ is the variation measure of the signed measure $x^{\prime} \nu(A)=\left\langle x^{\prime}, v(A)\right\rangle$, $A \in \Sigma, x^{\prime} \in X^{\prime}$. Observe that for all $A \in \Sigma$

$$
\sup \left\{p_{U}(\nu(B)), B \in \Sigma_{A}\right\} \leq\|v\|_{U}(A) \leq 2 \sup \left\{p_{U}(\nu(B)), B \in \Sigma_{A}\right\}
$$

where $\Sigma_{A}:=\{B \in \Sigma, B \subseteq A\}$; see [18, Lemma II.2].

Let $L^{1}(\nu, X)$ be the space of (classes of $v$-almost everywhere equal) scalar-valued integrable functions with respect to $\nu$. A real-valued, $\Sigma$-measurable function $f$ on $\Omega$ is called $\nu$-integrable (see $[18,19]$ ) if $f \in L^{\prime}\left(\left|x^{\prime} v\right|\right.$ ), for all $x^{\prime} \in X^{\prime}$, and if for each $A \in \Sigma$ there is a vector $\int_{A} f d v \in X$ (necessarily unique) satisfying $\left\langle x^{\prime}, \int_{A} f d \nu\right\rangle=\int_{A} f d\left(x^{\prime} \nu\right)$ for all $x^{\prime} \in X^{\prime}$. We identify two functions $f$ and $g$ if they are equal $\nu$-almost everywhere, that is, if

$$
\|v\|_{U}(\{\omega \in \Omega: f(\omega) \neq g(\omega)\})=0
$$

for all $U \in \mathscr{U}_{0}(X)$. The space $L^{1}(\nu, X)$ is a Fréchet lattice with the Lebesgue property when it is equipped with the topology of convergence in mean and the order $f \leq g$ if and only if $f(\omega) \leq g(\omega), v$-almost everywhere; see [19, Theorem 2.2] or [18, Corollary II.4.2]. Recall that a locally solid Riesz space $(L, \tau)$ is said to have the Lebesgue property (or that $\tau$ is a Lebesgue topology) if $u_{\alpha} \downarrow 0$ in $L$ implies $u_{\alpha} \stackrel{\tau}{\rightarrow} 0$. The characteristic function $\chi_{\Omega}$ is a weak order unit of the Fréchet lattice $L^{1}(\nu, X)$, since $\inf \left\{f, \chi_{\Omega}\right\}=0$ implies $f=0$. Moreover, a system of lattice seminorms for this topology is given by

$$
\|f\|_{U}:=\sup \left\{\int_{\Omega}|f| d\left|x^{\prime} v\right|, x^{\prime} \in U^{\circ}\right\}, \quad f \in L^{1}(v, X), U \in \mathscr{U}_{0}(X) .
$$

The associated integration map $I_{\nu}$ given by $I_{v}(f):=\int_{\Omega} f d v$ is linear and continuous from $L^{1}(\nu, X)$ into $X$.

In Section 2 we characterize the L-weakly compact sets of $L^{\prime}(\nu, X)$ via equiintegrability (Theorem 2.2). L-weak compactness of the range of a positive vector measure with values in a Fréchet lattice is also proved (Theorem 2.4). 
In Section 3 we consider the problem of relating the existence of copies of $\ell^{x}$ in the Fréchet space $L_{b}(Y, E)$, consisting of all linear continuous operators from a complete DF-space $Y$ to a Fréchet lattice $E$ (with the Lebesgue property) and having the topology of uniform convergence on the bounded sets of $Y$, with the coincidence of $L_{b}(Y, E)$ to a certain ideal of compact operators. Our results (Theorems 3.2 and 3.3) extend to the locally convex setting those of Curbera in [9]. Similar problems have been considered in $[4,5]$ and $[6]$.

In Section 4 we study sufficient conditions on the measure $v$ and the space $X$ in order that the space $L^{1}(v, X)$ has the Dunford-Pettis property (Theorem 4.1 and Corollary 4.2). Some applications to generalized Fréchet AL-spaces (Corollary 4.3) and Köthe spaces are also given; see Section 4 for the definition of these concepts.

Our notation and terminology is standard. For details concerning the lattice properties we refer the reader to $[20,21]$ and [23] and for the topological concepts in Riesz spaces to [1] and [2]. Aspects related to locally convex spaces can be seen in [15]. For the general theory of vector measures and integration we refer to the monographs [11] and [18].

\section{L-weakly compact sets in $L^{\prime}(v, X)$}

In this section we obtain a characterization of L-weakly compact sets in the space $L^{1}(\nu, X)$, where $X$ is a Fréchet space and $v: \Sigma \rightarrow X$ is a countably additive measure defined on a $\sigma$-algebra $\Sigma$ of subsets of a non-empty set $\Omega$. Recall (see [21, Definition 3.6.1]) that a (non-empty) subset $A$ of a Fréchet lattice $E$ is said to be L-weakly compact if $x_{n} \rightarrow 0$ in the topology of $E$ for every disjoint sequence $\left(x_{n}\right)_{n}$ contained in the solid hull $S(A)$ of $A$, where $S(A):=\{v \in E,|v| \leq|u|$ for some $u \in A\}$.

By using the disjoint sequence theorem of Aliprantis and Burkinshaw [1, Theorem 21.7] we can prove the following result; see also [21, Proposition 3.6.2].

THEOREM 2.1. Let $E$ be a Fréchet lattice and $K$ be a bounded subset of $E$. Then the following assertions are equivalent.

(1) $K$ is L-weakly compact.

(2) $x_{n}^{\prime}(x) \rightarrow 0$ uniformly on $K$ for every (positive) disjoint equi-continuous sequence $\left(x_{n}^{\prime}\right)_{n}$ in $E^{\prime}$.

Moreover, if $E$ has the Lebesgue property, then the above conditions are equivalent to

(3) $K$ is almost order bounded, that is, for every solid set $U \in \mathscr{U}_{0}(E)$ there exists $x \in E^{+}$such that $K \subset[-x, x]+U$. 
REMARK 2.1. In the course of this paper we will need the relationship between the concept of an L-weakly compact set and other notions of compactness. The position of the class of solid, bounded, L-weakly compact sets in a Fréchet lattice with the Lebesgue property, among other classes of compact sets, is given in the following items.

(1) L-weakly compact sets are relatively weakly compact, [1, Theorem 21.8]. The converse holds for generalized AL-spaces $E$. Indeed, $E$ is $|\sigma|\left(E, E^{\prime}\right)$-complete, [14, Theorem 1] and so $E=\left(E^{\prime}\right)_{n}^{\sim}$; see [1, Theorem 22.2]. Since $E^{\prime}$ is Dedekind complete [1, Theorem 5.7], it follows from Corollary 20.12 of [1], applied to $L=E^{\prime}$, that if a subset $A$ of $E$ is relatively weakly compact, then its convex solid hull is also relatively weakly compact. So, if $\left(x_{n}\right)_{n} \subseteq S(A)$ is pairwise disjoint, then also $\left(\left|x_{n}\right|\right)_{n} \subseteq S(A)$ is pairwise disjoint. By [1, Theorem 21.2] we see that $\left\langle\left|x^{\prime}\right|,\left|x_{n}\right|\right\rangle \rightarrow 0$ for each $x^{\prime} \in E^{\prime}$, that is, $x_{n} \rightarrow 0$ with respect to the absolute weak topology $|\sigma|\left(E, E^{\prime}\right)$ and hence, $x_{n} \rightarrow 0$ in $E$ by [14, Theorem 1]. Hence, $A$ is L-weakly compact.

(2) Solid, relatively compact sets are L-weakly compact, [1, Theorem 21.15]. The converse is true for discrete Fréchet lattices; see Theorems 21.12 and 21.15 of [1].

Suppose $X$ is a Fréchet space and let $v: \Sigma \rightarrow X$ be a vector measure. A positive measure $\lambda: \Sigma \rightarrow[0, \infty)$ is said to be a control measure for $v$ if $\lambda(A) \rightarrow 0, A \in \Sigma$ if and only if $\|v\|_{U}(A) \rightarrow 0$, for every $U \in \mathscr{U}_{0}(X)$. Let us observe that a control measure for a Fréchet valued measure always exists; see [18, II.1. Corollary 2 of Theorem 1].

THEOREM 2.2. Let $X$ be a Fréchet space, $v: \Sigma \rightarrow X$ be a countably additive measure and $K$ be a bounded subset of $L^{1}(\nu, X)$. Then the following assertions are equivalent.

(1) $K$ is L-weakly compact in $L^{1}(v, X)$.

(2) $\lim _{n}\left[\sup \left\{\left\|f \chi_{A_{n}}\right\|_{U}, f \in K\right\}\right]=0$, for every $U \in \mathscr{U}_{0}(X)$, and every sequence $\left(A_{n}\right)_{n} \downarrow \varnothing$ in $\Sigma$.

(3) $\lim _{\lambda(A) \rightarrow 0}\left[\sup \left\{\left\|f \chi_{A}\right\|_{U}, f \in K\right\}=0\right]$, for every $U \in \mathscr{U}_{0}(X)$, and every control measure $\lambda$ of $\nu$.

(4) $\lim _{\|\nu\|_{U}(A) \rightarrow 0}\left[\sup \left\{\left\|f \chi_{A}\right\|_{U}, f \in K\right\}\right]=0$, for every $U \in \mathscr{U}_{0}(X)$.

ProOF. (1) $\Rightarrow(2) \quad$ Take $U \in \mathscr{U}_{0}(X)$ and $\varepsilon>0$. Consider the solid neighborhood of 0 in $L^{1}(\nu, X)$ given by

$$
V_{\varepsilon}=\left\{f \in L^{1}(\nu, X):\|f\|_{U} \leq \frac{\varepsilon}{2}\right\}
$$

Since $K$ is L-weakly compact and $L^{1}(v, X)$ has the Lebesgue property, there exists $g_{\varepsilon}>0$ in $L^{1}(\nu, X)$ such that $K \subset\left[-g_{\varepsilon}, g_{\varepsilon}\right]+V_{\varepsilon}$; see Theorem 2.1. Hence, for every 
$f \in K$ we can write $f=u+v$, for some $v \in V_{\varepsilon}$ and $u$ with $|u| \leq g_{\varepsilon}$. Now, if $\left(A_{n}\right)_{n} \downarrow \varnothing$ in $\Sigma$ and $f \in K$, then

$$
\left\|f \chi_{A_{n}}\right\|_{U}=\left\|u \chi_{A_{n}}+v \chi_{A_{n}}\right\|_{U} \leq\left\||u| \chi_{A_{n}}\right\|_{U}+\frac{1}{2} \varepsilon \leq\left\|g_{\varepsilon} \chi_{A_{n}}\right\|_{U}+\frac{1}{2} \varepsilon .
$$

But, $g_{\varepsilon} \chi_{A_{n}} \rightarrow 0$ in $L^{1}(\nu, X)$, since $g_{\varepsilon} \chi_{A_{n}} \downarrow 0$ pointwise and $L^{1}(\nu, X)$ has the Lebesgue property, and so for some $N_{\varepsilon}$ we have that $\left\|g_{\varepsilon} \chi_{A_{n}}\right\|_{U} \leq \varepsilon / 2$ for all $n \geq N_{\varepsilon}$. It is then clear from (2.1) that (2) follows.

(2) $\Rightarrow$ (3) For every $f \in L^{1}(v, X)$ denote by $v_{f}: \Sigma \rightarrow X$ the measure $A \mapsto$ $\int_{A} f d \nu$. Take $U \in \mathscr{U}_{0}(X)$, and consider the following family of countably additive signed measures $\mathscr{M}:=\left\{x^{\prime} v_{f}: f \in K, x^{\prime} \in U^{\circ}\right\}$. This family is uniformly bounded with respect to the total variation norm, since $K$ is bounded in $L^{\prime}(v, X)$. By hypothesis, it is uniformly countably additive. Observe also that every member of $\mathscr{M}$ is $\lambda$-continuous. By $[11$, Corollary I.2.5], $\mathscr{M}$ is uniformly $\lambda$-continuous, that is,

$$
\lim _{\lambda(B) \rightarrow 0} \sup \left\{\left|x^{\prime} v_{f}(B)\right|, x^{\prime} \in U^{\circ}, f \in K\right\}=0 .
$$

Hence

$$
\lim _{\lambda(B) \rightarrow 0} \sup \left\{p_{U}\left(v_{f}(B)\right), f \in K\right\}=0
$$

and it follows that

$$
\lim _{\lambda(A) \rightarrow 0} \sup \left\{\left\|v_{f}\right\|_{U}(A), x^{\prime} \in U^{\circ}, f \in K\right\}=0,
$$

because $\left\|v_{f}\right\|_{U}(A) \leq 2 \sup \left\{p_{U}\left(v_{f}(B)\right), B \in \Sigma_{A}\right\}$. The conclusion then follows from the fact that

$$
\left\|v_{f}\right\|_{U}(A)=\left\|f \chi_{A}\right\|_{U},
$$

for all $A \in \Sigma$ and all $f \in K[19$, Theorem 2.2].

(3) $\Rightarrow$ (4) If $\lambda$ is any control measure for $\nu$, then $\lim _{\|v\|_{U}(A) \rightarrow 0} \lambda(A)=0$, for every $U \in \mathscr{U}_{0}(X)$ and so (4) follows from (3).

(4) $\Rightarrow$ (2) This is immediate since $\left(A_{n}\right)_{n} \downarrow \varnothing$ in $\Sigma$ implies that $\|v\|_{U}\left(A_{n}\right) \rightarrow 0$, for all $U \in \mathscr{U}_{0}(X)$; see [18, II.1. Lemma 3].

(3) $\Rightarrow$ (1) Let $\left(f_{n}\right)_{n}$ be a disjoint sequence in the solid hull of $K$. By definition of $S(K)$ there exist $g_{n} \in K$ such that $\left|f_{n}\right| \leq\left|g_{n}\right|$, for all $n=1,2, \ldots$ Consider the disjoint measurable sets $A_{n}:=\left\{\omega \in \Omega:\left|f_{n}(\omega)\right|>0\right\}, n=1,2, \ldots$, and observe that $\left|f_{n}\right| \chi_{A_{n}} \leq\left|g_{n}\right| \chi_{A_{n}}$, for all $n=1,2, \ldots$ Let $U \in \mathscr{U}_{0}(X)$ and $\varepsilon>0$. By the hypothesis (3) there exists $\delta>0$ such that

$$
\sup \left\{\left\|g \chi_{A}\right\|_{U}, g \in K\right\} \leq \varepsilon,
$$


for all $A \in \Sigma$ with $\lambda(A)<\delta$. To finish the proof observe that

$$
\left\|f_{n}\right\|_{U}=\left\|\left|f_{n}\right| \chi_{A_{n}}\right\|_{U} \leq\left\|\left|g_{n}\right| \chi_{A_{n}}\right\|_{U} \leq \sup \left\{\left\|g \chi_{A_{n}}\right\|_{U}, g \in K\right\} \leq \varepsilon,
$$

for $n$ large enough, because $\lim _{n} \lambda\left(A_{n}\right)=0$.

For $X$ a Banach space the following result can be found in [10, Claim 1, p. 3803].

COROLLARY 2.3. Let $X$ be a Fréchet space with the Schur property and let $\nu: \Sigma \rightarrow X$ be a countably additive measure. Then in $L^{1}(\nu, X)$ relatively weakly compact sets coincide with L-weakly compact sets.

PROOF. As we have already pointed out, every L-weakly compact set is relatively weakly compact. Suppose that there exists a set $K$ in $L^{1}(v, X)$ which is relatively weakly compact but is not L-weakly compact. By the condition (2) of Theorem 2.2, there exist $U \in \mathscr{U}_{0}(X)$, a sequence $\left(A_{n}\right)_{n} \downarrow \varnothing$ in $\Sigma$, and a sequence $\left(f_{n}\right)_{n} \subset K$ such that

$$
\left\|f_{n} \chi_{A_{n}}\right\|_{U} \geq \delta
$$

for some $\delta>0$ and all $n=1,2, \ldots$. Since $K$ is relatively weakly compact, by [15, Corollary 9.8.3] there exists a subsequence, that we still denote by $\left(f_{n}\right)_{n}$, which converges weakly in $L^{1}(\nu, X)$. Since $\left\|f \chi_{A}\right\|_{U} \leq\|f\|_{U}$, for all $U \in \mathscr{U}_{0}(X)$ and $A \in \Sigma$, the linear map $\Phi_{A}: f \mapsto f \chi_{A}$ is continuous from $L^{1}(\nu, X)$ into $L^{1}(\nu, X)$ for each $A \in \Sigma$. Hence, the composition map $I_{\nu} \circ \Phi_{A}: f \mapsto \int_{A} f d \nu$ is continuous from $L^{1}(\nu, X)$ into $X$. In particular, $I_{\nu} \circ \Phi_{A}$ is also continuous for the weak topologies on $L^{1}(\nu, X)$ and $X$. Accordingly, the sequence of integrals $\left(\int_{A} f_{n} d v\right)_{n}$ converges weakly in $X$ for every $A \in \Sigma$. Since $X$ is a Schur space, the convergence also holds in the topology of $X$. Let $v_{n}$ be the vector measure $A \mapsto \int_{A} f_{n} d \nu, A \in \Sigma$. These measures $v_{n}$ are countably additive and absolutely continuous with respect to any control measure $\lambda$ of $\nu$. Since $\left(\nu_{n}(A)\right)_{n}$ converges in $X$ for every $A \in \Sigma$, the Vitali-Hahn-Saks theorem implies that

$$
\lim _{\lambda(A) \rightarrow 0} \sup _{n} p_{V}\left(v_{n}(A)\right)=0
$$

for every $V \in \mathscr{U}_{0}(X)$. Then, we have

$$
\lim _{\lambda(A) \rightarrow 0} \sup _{n}\left\|f_{n} \chi_{A_{n}}\right\|_{V}=0,
$$

for every $V \in \mathscr{U}_{0}(X)$. But this is a contradiction of (2.2). 
We finish this section with an application of Theorem 2.2 to the properties displayed by the range of a vector-valued measure.

It is a classical result of Bartle, Dunford and Schwartz [3, Theorem 2.9] that the range of a vector measure with values in a Banach space is relatively weakly compact. This result was extended (in particular for Fréchet-valued measures) by Tweddle in [22] (see also [17, Theorem 2], [18, Theorem IV.6.1]). When the vector measure takes its values in a Fréchet lattice more can be said.

THEOREM 2.4. Let $E$ be a Fréchet lattice and let $v: \Sigma \rightarrow E$ be a positive countably additive measure. Then the solid hull of the range of $v$ is $L$-weakly compact.

PROOF. By using the condition (2) of Theorem 2.1 it is enough to show that $x_{n}^{\prime}(x) \rightarrow 0$ uniformly with respect to $x \in S(v(\Sigma))$, for every positive, disjoint and equi-continuous sequence $\left(x_{n}^{\prime}\right)_{n}$ in $E^{\prime}$. Now, for all $n=1,2, \ldots$ we have that

$$
\sup \left\{\left|x_{n}^{\prime}(x)\right|, x \in S(v(\Sigma))\right\} \leq \sup \left\{x_{n}^{\prime} \nu(A), A \in \Sigma\right\} \leq x_{n}^{\prime} \nu(\Omega)=x_{n}^{\prime} I_{v}\left(\chi_{\Omega}\right) .
$$

Indeed, the second inequality follows from the positivity of $x_{n}^{\prime}$ (given) and $I_{v}$ (easily verified). To verify the first inequality, note that if $x \in S(v(\Sigma))$, then $|x| \leq|y|$ for some $y \in v(\Sigma)$. Since $v$ is a positive measure $y=v(A)$ for some $A \in \Sigma$ and so $|y|=y$, that is, $|x| \leq v(A)$ for some $A \in \Sigma$. Since $x_{n}^{\prime}>0$ we have $x_{n}^{\prime}(|x|) \leq$ $x_{n}^{\prime}(v(A))$. But, $\left|x_{n}^{\prime}\right|=x_{n}^{\prime}$ and so, by [1, p. 21] we have $\left|x_{n}^{\prime}(x)\right| \leq x_{n}^{\prime}(|x|)$. Hence $\left|x_{n}^{\prime}(x)\right| \leq x_{n}^{\prime}(v(A))$ for some $A \in \Sigma$ whenever $x \in S(v(\Sigma))$, which establishes the first inequality. Since the order interval $\left[-\chi_{\Omega}, \chi_{\Omega}\right]$ is $\mathrm{L}$-weakly compact in $L^{1}(\nu, E)$, we conclude the proof by showing that the equi-continuous sequence of positive functionals $\left(x_{n}^{\prime} I_{v}\right)_{n}$ is disjoint. If $f>0$ in $L^{1}(v, E)$, then $u:=I_{v}(f) \geq 0$ in $E$. Therefore,

$$
\begin{aligned}
\inf \left\{x_{n}^{\prime} I_{v}, x_{m}^{\prime} I_{v}\right\}(f) & =\inf \left\{x_{n}^{\prime} I_{v}(g)+x_{m}^{\prime} I_{v}(f-g), 0 \leq g \leq f\right\} \\
& \leq \inf \left\{x_{n}^{\prime}(x)+x_{n}^{\prime}(u-x), x \in E, 0 \leq x \leq u\right\} \\
& =\inf \left\{x_{n}^{\prime}, x_{m}^{\prime}\right\}(u)=0 .
\end{aligned}
$$

REMARK 2.2. It is a well known fact that the unit ball of $\ell^{2}$ is the range of a vector measure [18, VII.4. Examples 1 and 2]. By considering the basis vectors $\left(e_{n}\right)_{n}$ it is clear that the unit ball of $\ell^{2}$ is a solid set which is not $\mathrm{L}$-weakly compact. This tells us that the statement of Theorem 2.4 is not true in general. Nevertheless, it still holds under a weaker hypothesis on the measure. A vector measure $\mu: \Sigma \rightarrow E$ is said to be dominated by a positive measure $\nu: \Sigma \rightarrow E$, if $|\mu(A)| \leq \nu(A)$, for all $A \in \Sigma$. In this case, the solid hull of the range of $\mu$ is obviously contained in the solid hull of 
the range of $\nu$. Thus, the solid hull of the range of $\mu$ is L-weakly compact, since any subset of a L-weakly compact set is also L-weakly compact. Observe that any vector measure $\mu$ with a Jordan decomposition ( $\mu=\mu_{1}-\mu_{2}$, with $\mu_{1}$ and $\mu_{2}$ positive vector measures) or any measure $\mu$ with a so called Hahn decomposition (that is, there exists $A \in \Sigma$ such that $\mu(B) \geq 0$ if $B \subseteq A$ and $\mu(B) \leq 0$ if $B \subseteq \Omega \backslash A$ ) is dominated by a positive measure.

\section{Operators with values in $L^{1}(\nu, X)$}

Let $Y$ be a complete DF-space (see [15, Section 12.4] for the definition) and let $X$ be a Fréchet space. Recall that $L_{b}(Y, X)$ denotes the Fréchet space (see [15, 12.4 Theorem 2]) of all linear continuous operators from $Y$ to $X$, equipped with the topology of uniform convergence on the bounded sets of $Y$. This topology is defined by the seminorms

$$
p_{U . H}(T):=\sup \left\{p_{U}(T y), y \in H\right\}, \quad T \in L_{b}(Y, X),
$$

where $U$ is any 0 -neighborhood in $X$ and $H$ is any bounded set in $Y$. In this section we extend to the locally convex case the results obtained by Curbera $[9$, Theorems 9 and 10] in the Banach case, about the existence of copies of $\ell^{\infty}$ in $L_{b}(Y, E)$ and its relationship to the coincidence of this space with some ideal of compact operators. Similar results have been proved in [5], [4] and [6] for pairs $(Y, X)$, where $X$ and $Y$ are either Fréchet or complete DF-spaces. To do this, we associate to each continuous linear operator $T: Y \rightarrow L^{1}(\nu, X)$ a vector measure taking values in the space of all linear continuous operators from $Y$ to $X$, and we characterize those operators whose associated measure is countably additive in the topology of uniform convergence on bounded sets of $Y$.

Consider the operator-valued set function $v_{T}: \Sigma \rightarrow L(Y, X)$, associated to the continuous linear operator $T: Y \rightarrow L^{1}(\nu, X)$ and the given vector measure $v: \Sigma \rightarrow$ $X$, which is defined by

$$
v_{T}(A): y \mapsto \int_{A} T y d v \in X \quad(y \in Y),
$$

that is, $v_{T}(A)=I_{\nu} \circ \Phi_{A} \circ T$ for each $A \in \Sigma$. It is then clear that $v_{T}$ is $L(Y, X)$-valued and finitely additive. Moreover, using (1.1) it can be shown that for every bounded set $H$ of $Y$ and each 0 -neighborhood $U$ of $X$, we have the following estimates for the $\|\cdot\|_{U, H}$-semivariation of $v_{T}$;

$$
\frac{1}{2} \sup \left\{\left\|T y \cdot \chi_{A}\right\|_{U}, y \in H\right\} \leq\left\|v_{T}\right\|_{U . H}(A) \leq 2 \sup \left\{\left\|T y \cdot \chi_{A}\right\|_{U}, y \in H\right\},
$$


for all $A \in \Sigma$. Moreover, it is easy to show that $\nu_{T}$ is countably additive in $L_{s}(Y, X)$, the space $L(Y, X)$ equipped with the topology of pointwise convergence. In general, $\nu_{T}$ is not countably additive in $L_{b}(Y, X)$; see [9, Example p. 322].

The following result can be found in [9, Theorem 4] for the case when both $X$ and $Y$ are Banach spaces.

THEOREM 3.1. Let $Y$ be a complete DF-space, $X$ be a Fréchet space, $v: \Sigma \rightarrow X$ be a countably additive measure and $T: Y \rightarrow L^{1}(\nu, X)$ be a continuous linear operator. The following conditions are equivalent.

(1) The operator $T$ is $L$-weakly compact, that is, $T$ maps bounded sets of $Y$ into $L$-weakly compact sets of the Fréchet lattice $L^{1}(\nu, X)$.

(2) The measure $v_{T}$ is strongly additive in $L_{b}(Y, X)$, that is, $v_{T}\left(A_{n}\right) \rightarrow 0$ in $L_{b}(Y, X)$ whenever $\left(A_{n}\right)_{n}$ is a disjoint sequence in $\Sigma$.

(3) The measure $v_{T}$ is countably additive in $L_{b}(Y, X)$.

PROOF. (1) $\Rightarrow(2) \quad$ Suppose that $v_{T}$ is not strongly additive. By the Fréchet space version of [11, Corollary I.1.18] there exist a bounded set $H$ in $Y$, a 0 -neighborhood $U$ in $X$, a pairwise disjoint sequence of measurable sets $\left(A_{n}\right)_{n}$ and an $\varepsilon>0$ such that $\left\|v_{T}\right\|_{U . H}\left(A_{n}\right) \geq \varepsilon>0$, for all $n=1,2, \ldots$ By using the bounds given for the semivariations of the measure $v_{T}$ in (3.1) we can choose a $y_{n} \in H$ such that $\left\|T y_{n} \cdot \chi_{A_{n}}\right\|_{U} \geq \varepsilon / 2$, for each $n=1,2, \ldots$ But this contradicts (1), since $\left(T y_{n} \cdot \chi_{A_{n}}\right)_{n}$ is then a disjoint sequence in the solid hull of $T(H)$ that does not converge to 0 .

(2) $\Rightarrow$ (1) Suppose that $T$ is not $\mathrm{L}$-weakly compact. Then there exists a bounded set $H$ in $Y$ such that $T(H) \subset L^{1}(\nu, X)$ is not L-weakly compact. Then we can take a positive and disjoint sequence $\left(f_{n}\right)_{n}$ in $L^{1}(\nu, X)$ such that $f_{n} \leq\left|T y_{n}\right|$ for certain $y_{n} \in H(n=1,2, \ldots)$ but $\left(f_{n}\right)_{n}$ does not converge to 0 . By passing to a subsequence, there exists $U \in \mathscr{U}_{0}(X)$ such that $\left\|f_{n}\right\|_{U} \geq 1$, for all $n=1,2, \ldots$. Consider the disjoint sequence $\left(A_{n}\right)_{n}$ of measurable sets $A_{n}:=\left\{\omega \in \Omega: f_{n}(\omega)>0\right\}$. Then (3.1) implies that

$$
\begin{aligned}
1 & \leq\left\|f_{n}\right\|_{U} \leq\left\|\left|T y_{n}\right| \cdot \chi_{A_{n}}\right\|_{U}=\left\|\left|T y_{n} \cdot \chi_{A_{n}}\right|\right\|_{U}=\left\|T y_{n} \cdot \chi_{A_{n}}\right\|_{U} \\
& \leq \sup \left\{\left\|T y \chi_{A_{n}}\right\|_{U}, \quad y \in H\right\} \leq 2\left\|\nu_{T}\right\|_{U, H}\left(A_{n}\right),
\end{aligned}
$$

for all $n=1,2, \ldots$ Once again, by [11, Corollary I.1.18], $v_{T}$ is not strongly additive.

(2) $\Rightarrow$ (3) Since the measure $v_{T}: \Sigma \rightarrow L_{b}(Y, X)$ is countably additive in a weaker Hausdorff topology (that is, in $L_{s}(Y, X)$ ) it is routine to check that the strong additivity of $v_{T}$ in $L_{b}(Y, X)$ implies its countable additivity.

(3) $\Rightarrow$ (2) This is obvious.

For the case when $Y$ is a Banach space and $E$ is a Banach lattice with order continuous norm and a weak order unit, the following result can be found in $[9$, Theorem 9]. 
THEOREM 3.2. Let $E$ be a Fréchet lattice with the Lebesgue property and with a weak order unit and let $Y$ be a complete DF-space. If $L_{b}(Y, E)$ does not contain an isomorphic copy of $\ell^{\infty}$, then every continuous linear operator $T$ from $Y$ to $E$ is L-weakly compact.

Proof. By the representation theorem [12, Proposition 2.4 (vi)] there exists a measurable space $(\Omega, \Sigma)$ and a countably additive vector measure $v: \Sigma \rightarrow E$ such that $E$ is lattice isomorphic to $L^{1}(\nu, E)$. Thus, we can consider the operator $T$ as mapping $Y$ into $L^{1}(\nu, E)$. Then the associated measure $v_{T}$ takes its values in $L(Y, E)$ and has bounded range in $L_{b}(Y, E)$. By a theorem of Diestel and Faires [8, Corollary 4.1.44 and Theorem 4.7.16] the measure $v_{T}$ is strongly additive. Accordingly, the operator $T$ is L-weakly compact by Theorem 3.1 .

Recall that a Fréchet lattice is said to be discrete if there exists a complete disjoint system of atoms. (See [1, p. 17 and Example 9, p. 31].) In this setting, we know that L-weakly compact sets are relatively compact; see Remark 2.1 . The theorem to follow is an extension of part of [9, Theorem 10] (a similar result to [16, Theorem 6], without restrictions in the first space). For its proof we will need the following lemma which, in the Banach space case, is contained in the proof of $[9$, Theorem 10]. We include it for the sake of completeness.

LEMMA 3.1. Let $E$ be a Fréchet lattice with the Lebesgue property. Let $A(E)$ be a maximal disjoint system of positive atoms in $E$. For every $x \in E$ the set $A(x):=\{z \in A(E), \inf \{z,|x|\} \neq 0\}$ is countable.

PROOF. Since $E$ has the Lebesgue property it is Dedekind complete, [1, Theorem 10.3]. Hence the order projection $P_{z}$ associated with the element $z \in E$ exists, [1, Theorem 2.11], and satisfies

$$
P_{z}(v)=\sup \{\inf \{v, n|z|\}: n \in \mathbb{N}\}, \quad v \in E^{+}
$$

see [1, p. 13]. Fix any $x \in E$. For every $\varepsilon>0$ and every continuous lattice seminorm $q$, the set $\left\{z \in A(E), q\left(P_{z}(|x|)\right) \geq \varepsilon\right\}$ is finite. If this is not the case, we can find an infinite sequence of atoms $\left(z_{n}\right)_{n}$ from $A(E)$ such that $q\left(P_{z_{n}}(|x|)\right) \geq \varepsilon$, for all $n=1,2, \ldots$ Consider the increasing sequence $u_{k}:=P_{z_{1}+\ldots+z_{k}}(|x|), k=1,2, \ldots$ This sequence is order bounded by $|x|$. Since $E$ has the Lebesgue property, $\left(u_{k}\right)_{k}$ must be convergent. But, it follows from (3.2) that

$$
\left.q\left(u_{k}-u_{k-1}\right)=q\left(P_{z_{k}}(|x|)\right)\right) \geq \varepsilon \quad \text { for all } k=2,3, \ldots
$$

which is a contradiction. 
Now, consider an increasing sequence $\left(q_{n}\right)_{n}$ of lattice seminorms generating the topology of $E$. Then it can be shown that

$$
A(x)=\bigcup_{n . m=1}^{\infty}\left\{z \in A(E), q_{n}\left(P_{z}(|x|)\right) \geq \frac{1}{m}\right\}
$$

and this set is countable.

THEOREM 3.3. Let $E$ be a discrete Fréchet lattice with the Lebesgue property and $Y$ be a complete DF-space. If $L_{b}(Y, E)$ does not contain an isomorphic copy of $\ell^{\infty}$, then every continuous linear operator from $Y$ to $E$ is compact.

PROOF. If $Y$ is a DF-space and $X$ a Fréchet space, then the compact operators from $Y$ to $X$ coincide with Montel operators from $Y$ to $X$. (See the remark (1) after [5, Corollary 19].) Recall that a continuous linear map from $Y$ to $X$ is called Montel if it transforms bounded sets into relatively compact sets.

If $E$ has a weak order unit, bearing in mind (by Remark 2.1 (2)) that L-weakly compact sets are relatively compact, it follows that every operator from $Y$ to $E$ is Montel, by the previous paragraph and Theorem 3.2. Now consider the general case (that is, no weak order unit) and suppose that there exists a continuous linear operator $T: Y \rightarrow E$ which is not Montel. Then there exist a bounded sequence $\left(x_{n}\right)_{n}$ in $Y$, and $U \in \mathscr{U}_{0}(E)$ such that

$$
p_{U}\left(T x_{n}-T x_{m}\right) \geq 1, \text { for all } n \neq m
$$

By Lemma 3.1 , the set $H:=\bigcup_{n \geq 1} A\left(T x_{n}\right)$ is countable. The band $F$ generated by $H$ coincides with the subspace generated by $H$, since all of the elements of $H$ are atoms. If we consider on $F$ its relative topology, it is a discrete Fréchet lattice with the Lebesgue property. Moreover it has a weak order unit, since it is separable [1, Example 7 p. 123]. On the other hand, $T x_{n} \in F$, for all $n=1,2, \ldots$ since $\inf \left\{\left|T x_{n}\right|, z\right\}=0$, for all $z \notin A\left(T x_{n}\right), n=1,2, \ldots$ Denote by $P_{F}: E \rightarrow F$ the order projection band onto $F$. Then $P_{F} T \in L(Y, F)$ and is not compact by (3.3) as $p_{U}\left(P_{F} T x_{n}-P_{F} T x_{m}\right)=p_{U}\left(T x_{n}-T x_{m}\right)$. Moreover $L_{b}(Y, F)$ does not contain an isomorphic copy of $\ell^{\infty}$ as it is a closed subspace of $L_{b}(Y, E)$. But, the previous case shows that $P_{F} T$ is Montel and so we have a contradiction.

REMARK 3.1. The converse of the above theorem is not true in general. (See the remark after the corollary below.) Nevertheless, it is true if the discrete Fréchet lattice $E$ is non-Montel, in addition to having the Lebesgue property. Suppose that $L_{b}(Y, E)=M_{b}(Y, E)$ has a copy of $\ell^{\infty}$. According to [5, Corollary 19 (a)], $Y$ contains a complemented copy of $\ell^{1}$ or $E$ contains a copy of $\ell^{\infty}$. The latter case is 
impossible, because $E$ has the Lebesgue property [1, Theorem 10.7]. If we take a bounded sequence $\left(x_{n}\right)_{n}$ in $E$ without convergent subsequences (which is possible as $E$ is non-Montel), then the operator $T(\alpha):=\sum_{n} \alpha_{n} x_{n}$, for $\alpha \in \ell^{1}$, defines a nonMontel operator from $\ell^{1}$ to $E$. It is then possible to construct a non-Montel operator from $Y$ to $E$ and a contradiction follows.

Since no infinite dimensional Banach space is Montel, we point out that Remark 3.1 and Theorem 3.3 together are an extension of (all of) [9, Theorem 10].

We complete this section with an application to Köthe spaces. Compare our result with [6, Theorem 3] and [5, Propositions 29 and 30]. Consider an index set $I$, not assumed to be countable. Recall that an increasing sequence $A=\left(a_{k}\right)_{k}$ of positive families $a_{k}=\left(a_{k i}\right)_{i \in l}$ is called a Köthe matrix if for each $i \in I$ there exists a $k \geq 1$ such that $a_{k i}>0$. For $1 \leq p<\infty$ we define

$$
\lambda_{p}(I, A):=\left\{x=\left(x_{i}\right)_{i \in I}:\|x\|_{k}^{p}:=\left(\sum_{i \in I}\left|x_{i}\right|^{p} a_{k i}\right)^{l / p}<\infty, k=1,2, \ldots\right\}
$$

equipped with the topology generated by the seminorms $\|\cdot\|_{k}^{p}, k=1,2, \ldots$ Then $\lambda_{p}(I, A)$, called a Köthe space, is a discrete Fréchet lattice with the Lebesgue property which has a weak order unit if and only if the index set $I$ is countable. To check that $\lambda_{p}(I, A)$ has the Lebesgue property use the $p$-additivity property of each seminorm $\|\cdot\|_{k}^{p}, k=1,2, \ldots$ as in $[1$, Theorem 10.10].

COROLlary 3.4. Let $\lambda_{p}(I, A)$ be a Köthe space and $Y$ be a complete DF-space. If $L_{b}\left(Y, \lambda_{p}(I, A)\right)$ does not contain an isomorphic copy of $\ell^{\infty}$, then every continuous linear operator from $Y$ to $\lambda_{p}(I, A)$ is compact (or Montel).

REMARK 3.2. Equivalent conditions under which $L_{b}\left(Y, \lambda_{p}(I, A)\right)$ has a copy of $\ell^{\infty}$, for a Fréchet or a complete DF-space $Y$ and $2 \leq p<\infty$ have been studied in [4, Corollary 21 ].

\section{Operators defined on $L^{1}(v, X)$}

Let $X$ be a Fréchet space and $v: \Sigma \rightarrow X$ be a vector measure of bounded variation. That is, for every 0 -neighborhood $U$ on $X$, we have

$$
|v|_{U}(\Omega):=\sup _{\pi} \sum_{A \in \pi} p_{U}(\nu(A))<\infty,
$$

where the supremum is taken over all partitions $\pi$ of $\Omega$. For technical reasons we will require that

$$
D_{v}:=\left\{x^{\prime} \in X^{\prime}: v \text { is }\left|x^{\prime} v\right| \text {-continuous }\right\} \neq \varnothing,
$$


where to say that $v$ is $\lambda$-continuous with respect to a positive measure $\lambda$ on $\Sigma$ means that every $\lambda$-null set is a $\nu$-null set. Measures of the form $\left|x^{\prime} v\right|$ with $x^{\prime} \in D_{\nu}$ (when they exist) are called Rybakov control measures for $\nu$. Conditions on the space $X$ for which (4.1) holds have been studied in [13], where it is shown that if $X$ admits a continuous norm, then every $X$-valued vector measure has a Rybakov control measure. In this section we study sufficient conditions on $v$ and $X$ in order that the space $L^{1}(v, X)$ has the Dunford-Pettis property. Recall that a Fréchet space is said to have the DunfordPettis property if every weakly compact operator defined on it maps relatively weakly compact sets into relatively compact sets. We also apply these results to the class of Fréchet AL-spaces with a continuous norm.

We first establish some preparatory results concerning the representation of operators from $L^{1}(\nu, X)$ to $Y$. The next lemma is the vector version of [13, Lemma 3.1 (B)].

LEMMA 4.1. Let $X$ and $Y$ be Fréchet spaces, $v: \Sigma \rightarrow X$ be a countably additive vector measure and $T: L^{1}(\nu, X) \rightarrow Y$ be a continuous linear map. Then the map $\mu_{T}: \Sigma \rightarrow Y$, given by $\mu_{T}(A):=T\left(\chi_{A}\right)$, defines a countably additive vector measure (henceforth called the representing measure for $T$ ), the inclusion $L^{1}(\nu, X) \subset$ $L^{1}\left(\mu_{T}, Y\right)$ holds in the sense of vector spaces, and

$$
T(f)=\int_{\Omega} f d \mu_{T} \quad\left(f \in L^{1}(\nu, X)\right) .
$$

ProOF. The formula $\mu_{T}(A):=T\left(\chi_{A}\right)$ defines a finitely additive measure $\mu_{T}$ : $\Sigma \rightarrow Y$. Actually, this measure is countably additive by the dominated convergence theorem for vector measures [18, Theorem II.4.2] and the continuity of $T$. To prove the inclusion $L^{1}(\nu, X) \subset L^{1}\left(\mu_{T}, Y\right)$ it suffices to show that every non-negative function $f \in L^{1}(\nu, X)$ belongs to $L^{1}\left(\mu_{T}, Y\right)$. To see this, choose a sequence of $\Sigma$-measurable simple functions $0 \leq \varphi_{1} \leq \varphi_{2} \leq \cdots \leq f$ which increases pointwise to $f$ on $\Omega$. By the dominated convergence theorem for vector measures we have $\chi_{A} \varphi_{n} \rightarrow \chi_{A} f$ in $L^{1}(\nu, X)$, for each $A \in \Sigma$. The continuity of $T$ implies that $T\left(\chi_{A} \varphi_{n}\right) \rightarrow T\left(\chi_{A} f\right)$ in $Y$ as $n \rightarrow \infty$. That is, the sequence $T\left(\chi_{A} \varphi_{n}\right)=\int_{A} \varphi_{n} d \mu_{T}, n=1,2, \ldots$ is convergent in $Y$ for each $A \in \Sigma$. Now, since $\nu$-null sets are $\mu_{T}$-null sets, it follows from [19, Lemma 2.3] that $f \in L^{1}\left(\mu_{T}, Y\right)$. By the dominated convergence theorem for $\mu_{T}$ it is also follows (now knowing that $f \in L^{1}\left(\mu_{T}, Y\right)$ ) that $\int_{\Omega} \varphi_{n} d \mu_{T} \rightarrow \int_{\Omega} f d \mu_{T}$, and this establishes that $\int_{\Omega} f d \mu_{T}=T(f)$.

For the notions of strongly $\lambda$-measurable and $\lambda$-integrable functions $f: \Omega \rightarrow X$ (with $X$ a Fréchet space) with respect to a finite positive measure $\lambda$ we refer to [7], for example. The function $f$ is called $\lambda$-Pettis integrable if $\left\langle x^{\prime}, f\right\rangle \in L^{1}(\lambda)$, for all $x^{\prime} \in X^{\prime}$, and for each $A \in \Sigma$ there is a vector $\int_{A} f d \lambda \in X$ such that $\left\langle x^{\prime}, \int_{A} f d \lambda\right\rangle=\int_{A}\left\langle x^{\prime}, f\right\rangle d \lambda$ 
for all $x^{\prime} \in X^{\prime}$. For $X$ and $Y$ Banach spaces the following result can be found [10, p. 3804]; see the proof of Claim 2 given there.

LEMMA 4.2. Let $X$ and $Y$ be Fréchet spaces, let $\nu: \Sigma \rightarrow X$ be a countably additive vector measure of bounded variation, and let $T: L^{1}(v, X) \rightarrow Y$ be a weakly compact operator. Then, for every control measure $\lambda: \Sigma \rightarrow[0,+\infty)$ of $\nu$, the representing measure $\mu_{T}$ has a Radon-Nikodym derivative with respect to $\lambda$, that is, there exists a strongly $\lambda$-measurable function $g: \Omega \rightarrow Y$ which is $\lambda$-integrable such that

$$
\mu_{T}(A)=\int_{A} g d \lambda, \quad \text { for all } A \in \Sigma .
$$

Moreover, for every function $f \in L^{1}(\nu, X)$, the function $f g$ is strongly $\lambda$-measurable and $\lambda$-Pettis integrable and its Pettis integral $\int_{\Omega} f g d \lambda$ satisfies

$$
T(f)=\int_{\Omega} f g d \lambda .
$$

ProOF. By using the continuity of $T$ and the fact that $\nu$ has bounded variation we can see that the measure $\mu_{T}$ has bounded variation. Moreover, $\mu_{T}$ is $\lambda$-continuous and has locally relatively weakly compact (hence, s-dentable by [7, Theorem 1.1]) average range, meaning that for every $A \in \Sigma^{+}$there exists $B \in \Sigma^{+}, B \subseteq A$, such that

$$
\mathscr{R}_{B}\left(\mu_{T}\right):=\left\{\frac{\mu_{T}(C)}{\lambda(C)}, C \in \Sigma^{+}, \text {and } C \subseteq B\right\}
$$

is relatively weakly compact, where $\Sigma^{+}=\{A \in \Sigma, \lambda(A)>0\}$. To see this, observe that $\mu_{T}(C) / \lambda(C)=T\left(\chi_{C} / \lambda(C)\right)$ for all $C \in \Sigma^{+}$, so that $\mathscr{R}_{B}\left(\mu_{T}\right)=T\left(\mathscr{R}_{B}(\mu)\right)$, for all $B \in \Sigma^{+}$, where $\mu: \Sigma \rightarrow L^{1}(\nu, X)$ is the countably additive $\lambda$-continuous vector measure of bounded variation given by $\mu(A):=\chi_{A}$. With this observation, and recalling that $T$ is weakly compact, it is enough to show that $\mu$ has locally bounded average range in $L^{1}(v, X)$. But, this follows from [7, Lemma 3.1]. Now, by [7, Theorem 2.1$]$ there exists a strongly $\lambda$-measurable and $\lambda$-integrable function $g: \Omega \rightarrow Y$ (called Bochner integrable in the Banach space case) such that

$$
\mu_{T}(A)=\int_{A} g d \lambda, \quad A \in \Sigma
$$

Moreover,

$$
\left\langle y^{\prime}, \mu_{T}(A)\right\rangle=\int_{A}\left\langle y^{\prime}, g\right\rangle d \lambda, \quad A \in \Sigma, y^{\prime} \in Y^{\prime}
$$


Now, from Lemma 4.1 and (4.4), we can prove that $\left\langle y^{\prime}, f g\right\rangle \in L^{\prime}(\lambda)$ for all $f \in$ $L^{1}(\nu, X)$ and all $y^{\prime} \in Y$. Moreover,

$$
\left\langle y^{\prime}, \int_{A} f d \mu_{T}\right\rangle=\int_{A} f d\left(y^{\prime} \mu_{T}\right)=\int_{A} f\left\langle y^{\prime}, g\right\rangle d \lambda=\int_{A}\left\langle y^{\prime}, f g\right\rangle d \lambda .
$$

Hence $f g: \Omega \rightarrow Y$ is $\lambda$-Pettis integrable and its Pettis integral is $\int_{A} f g d \lambda=\int_{A} f d \mu_{T}$, for all $A \in \Sigma$. This together with (4.2) gives (4.3).

Finally, to see that $f g$ is strongly $\lambda$-measurable note that $g$ takes its values in a separable subspace of $Y$ and hence, so does $f g$. Clearly $\left\langle y^{\prime}, f g\right\rangle$ is $\Sigma$-measurable for each $y^{\prime} \in Y^{\prime}$ and so $f g: \Omega \rightarrow Y$ is scalarly measurable. Then the Pettis measurability theorem (which is also valid in Fréchet spaces) implies that $f g$ is strongly $\lambda$-measurable.

For Banach spaces the following result occurs in [10, Claim 2, p. 3804].

THEOREM 4.1. Let $X$ and $Y$ be Fréchet spaces and let $v: \Sigma \rightarrow X$ be a vector measure of bounded variation for which $D_{v} \neq \varnothing$. If $T: L^{1}(\nu, X) \rightarrow Y$ is a weakly compact operator, then $T$ maps $L$-weakly compact sets into relatively compact sets.

PROOF. Let $\lambda$ be a Rybakov control measure for $\nu$. By Lemma 4.2, there is a strongly $\lambda$-measurable and $\lambda$-integrable function $g: \Omega \rightarrow Y$, such that

$$
T(f)=\int_{\Omega} f g d \lambda, \quad f \in L^{1}(\nu, X) .
$$

Let $K$ be an L-weakly compact (solid) set in $L^{1}(\nu, X)$. To see that $T(K)$ is a relatively compact subset of $Y$ it is enough to show (see [2, Theorem 9.1] and [15, Theorem 3.5.1]) that for every $V \in \mathscr{U}_{0}(Y)$ there exists a relatively compact set $K_{V} \subset Y$ such that $T(K) \subset K_{V}+V$. So, fix a 0 -neighborhood $V$ in $Y$.

First of all, observe that $\left\{\int_{\Omega}|f| d \lambda, f \in K\right\}$ is a bounded set, since $\lambda$ is a Rybakov control measure for $v$ and $K$ is an L-weakly compact set (hence bounded by Remark $2.1(1))$ in $L^{1}(\nu, X)$. Then we can put $\rho:=\sup \left\{\int_{\Omega}|f| d \lambda, f \in K\right\}$.

From the continuity of $T$ there exists a 0 -neighborhood $U$ in $X$ such that $p_{V}(T f) \leq$ $\|f\|_{U}$, for all $f \in L^{1}(\nu, X)$.

By Theorem 2.2, we have that $\lim _{\lambda(A) \rightarrow 0} \sup \left\{\left\|f \chi_{A}\right\|_{U}, f \in K\right\}=0$ and so there exists $\delta>0$ such that $\sup \left\{\left\|f \chi_{A}\right\|_{U}, f \in K\right\}<\frac{1}{2}$, for all $A \in \Sigma$ with $\lambda(A)<\delta$.

Since the function $g$ is strongly $\lambda$-measurable, by Egoroff's theorem there exist a $\Sigma$-simple function $\varphi: \Omega \rightarrow Y$ and a set $B \in \Sigma$ with $\lambda(B)<\delta$ such that

$$
p_{V}(g(\omega)-\varphi(\omega)) \leq \frac{1}{2 \rho}, \quad \omega \in \Omega \backslash B
$$


For $f$ in $K$ we have

$$
T(f)=T\left(f \chi_{B}\right)+\int_{\Omega \backslash B} f \varphi d \lambda+\int_{\Omega \backslash B} f(g-\varphi) d \lambda
$$

Then

$$
\begin{aligned}
p_{V}\left(T(f)-\int_{\Omega \backslash B} f \varphi d \lambda\right) & \leq p_{V}\left(T\left(f \chi_{B}\right)\right)+p_{V}\left(\int_{\Omega \backslash B} f(g-\varphi) d \lambda\right) \\
& \leq\left\|f \chi_{B}\right\|_{U}+\int_{\Omega \backslash B}|f| p_{V^{\prime}}(g-\varphi) d \lambda \\
& \leq \frac{1}{2}+\rho \frac{1}{2 \rho}=1 .
\end{aligned}
$$

Hence $T(K) \subset\left\{\int_{\Omega \backslash B} f \varphi d \lambda, f \in K\right\}+V$. Finally, the set

$$
K_{V}:=\left\{\int_{\Omega \backslash B} f \varphi d \lambda, f \in K\right\}
$$

is relatively compact in $Y$ because $\varphi$ is a $\Sigma$-simple function and $K$ is bounded.

For the case when $X$ is a Banach space the following result occurs in [10, Theorem 41.

COROLlARY 4.2. Let $X$ be a Fréchet space with the Schur property and let $v$ : $\Sigma \rightarrow X$ be a vector measure of bounded variation for which $D_{v} \neq \varnothing$. Then the space $L^{\prime}(\nu, X)$ has the Dunford-Pettis property.

ProOF. The result follows from Corollary 2.3 and Theorem 4.1.

We conclude this section by showing that an important class of Fréchet lattices, the so called generalized AL-spaces, have the Dunford-Pettis property. Such spaces have been studied intensively in [14, Section 2], A Fréchet lattice $E$ is a generalized AL-space if its topology can be defined by a family of lattice seminorms $p$ that are additive on the positive cone, that is, with $p(x+y)=p(x)+p(y)$, for $x, y \in E^{+}$.

We recall at this point (see Section 3) that a Köthe space $\lambda_{1}(I, A)$ is a generalized AL-space with a weak order unit if and only if the index set $I$ is countable. Moreover it has a continuous norm if one of the steps, say $a_{k}=\left(a_{k i}\right)_{i \in I}$, is strictly positive.

COROLLARY 4.3. Let $E$ be a generalized AL-Fréchet space with a weak order unit and a continuous norm. Then $E$ has the Dunford-Pettis property. 
ProOF. Since every AL-space has the Lebesgue property (see Section 3), according to the representation theorem [12, Proposition 2.4 (vi)], $E$ is lattice isomorphic to $L^{1}(\nu, E)$, for a certain countably additive measure $v: \Sigma \rightarrow E$. Hence, every operator defined on $E$ can be considered as being defined on $L^{\prime}(\nu, E)$. Moreover, an examination of the proof of [12, Proposition 2.4 (vi)] (see also p. 364 there) shows that $v(\Sigma) \subseteq E^{+}$. So, if $p_{U}$ is a lattice seminorm for $E$ which is additive on $E^{+}$, then it is routine to verify that $\sum_{A \in \pi} p_{U}(\nu(A))=p_{U}(\nu(\Omega))$ for every partition $\pi$ of $\Omega$. Accordingly, $|v|_{U}(\Omega) \leq p_{U}(v(\Omega))<\infty$ which shows that $\nu$ has bounded variation. Since $E$ has a continuous norm, $D_{v} \neq \varnothing$, that is, the measure $v$ has a Rybakov control measure [13, Theorem 2.2]. Note that the relatively weakly compact sets coincide with L-weakly compact sets in $L^{1}(\nu, E)$, provided that $L^{1}(\nu, E)$ is an AL-space; see Remark 2.1(1). The proof then follows by applying Theorem 4.1. So, it remains to establish that $L^{1}(v, E)$ is a generalized AL-space whenever $E$ is a generalized AL-Fréchet space and $v: \Sigma \rightarrow E$ is a positive measure (necessarily having bounded variation). The following lemma establishes this fact.

LEMMA 4.3. Let $E$ be a generalized AL-Fréchet space and $v: \Sigma \rightarrow E$ be a positive measure. Then $L^{1}(\nu, E)$ is a generalized AL-space.

PROOF. Denote by $\mathscr{U}_{0}(E)$ the system of all solid 0 -neighborhoods in $E$. Since $E$ is a generalized AL-Fréchet space its topology is $|\sigma|\left(E, E^{\prime}\right)$; see [14, Theorem 1]. Now, by applying [2, Theorem 11.11(1)] we have that for every $U \in \mathscr{U}_{0}(E)$ there exists $x_{U}^{\prime}>0$ in $E^{\prime}$ such that $U^{\circ} \subseteq\left[-x_{U}^{\prime}, x_{U}^{\prime}\right]$, that is, $\left|x^{\prime}\right| \leq x_{U}^{\prime}$, for all $x^{\prime} \in U^{\circ}$. Observe that

$$
\left|x^{\prime} v\right|(A) \leq x_{U}^{\prime} v(A), \quad x^{\prime} \in U^{\circ}, A \in \Sigma
$$

Consider the following system of lattice seminorms $\left(x_{U}^{\prime} v\right.$ is a positive measure) on $L^{1}(\nu, E)$ :

$$
|f|_{U}:=\int_{\Omega}|f| d\left(x_{U}^{\prime} v\right), \quad f \in L^{1}(v, E)
$$

where $U \in \mathscr{U}_{0}(E)$. Now, it is clear from (4.6) that this system of seminorms define the topology of $L^{1}(\nu, E)$. Moreover, $|f+g|_{U}=|f|_{U}+|g|_{U}$, for all positive functions $f, g \in L^{1}(\nu, E)$ and every $U \in \mathscr{U}_{0}(E)$. This shows that $L^{1}(\nu, E)$ is a generalized AL-space.

The authors thank the referee for a number of suggestions and comments which improve the content and presentation of the paper. 


\section{References}

[1] C. D. Aliprantis and O. Burkinshaw, Locally solid Riesz spaces, Pure Appl. Math., Vol. 76 (Academic Press, Orlando, Florida, 1978).

[2] - Positive operators, Pure Appl. Math., Vol. 119 (Academic Press, Orlando, Florida, 1985).

[3] R. G. Bartle, N. S. Dunford and J. T. Schwartz, 'Weak compactness and vector measures', Canad. J. Math. 7 (1955), 289-305.

[4] J. Bonet, P. Domański and M. Lindström, 'Cotype and complemented copies of $c_{0}$ in spaces of operators', Czechoslovak Math. J. 46 (1996), 271-289.

[5] J. Bonet, P. Domański, M. Lindström and M. S. Ramanujan, 'Operator spaces containing $c_{0}$ or $\ell^{\infty}$, Resultate Math. 28 ( 1995 ), 250-269.

[6] J. Bonet and M. Lindström, 'Spaces of operators between Fréchet spaces', Math. Proc. Camb. Phil. Soc. 115 (1994), 133-144.

[7] G. Y. H. Chi, 'A geometric characterization of Fréchet spaces with the Radon-Nikodým property', Proc. Amer. Math. Soc. 48 (1975), 371-380.

[8] C. Constantinescu, Spaces of measures, Studies in Mathematics 4 (de Gruyter, Berlin, New York, 1984).

[9] G. P. Curbera, 'Operators into $L^{1}$ of a vector measure and applications to Banach lattices', Math. Ann. 292 (1992), 317-330.

[10] _ 'Banach space properties of $L^{\prime}$ of a vector measure', Proc. Amer: Math. Soc. 123 (1995), $3797-3806$.

[11] J. Diestel and J. J. Uhl, Vector measures, Math. Surveys Monographs, Vol. 15 (Amer. Math. Soc., Providence, RI, 1977).

[12] P. G. Dodds, B. de Pagter and W. J. Ricker, 'Reflexivity and order properties of scalar-type spectral operators in locally convex spaces', Trans. Amer. Math. Soc. 293 (1986), 355-380.

[13] A. Fernández and F. Naranjo, 'Rybakov's theorem for vector measures in Fréchet spaces', Indag. Math. (N.S.) 8 (1997), 33-42.

[14] K. G. Grosse-Erdmann, 'Lebesgue's theorem of differentiation in Fréchet lattices', Proc. Amer: Math. Soc. 112 (1991), 371-379.

[15] H. Jarchow, Locally convex spaces (Teubner, Stuttgart, 1981).

[16] N. Kalton, 'Spaces of compact operators', Math. Ann. 208 (1974), 267-278.

[17] I. Kluvánek, 'Characterization of the closed convex hull of the range of a vector-valued measure', J. Funct. Anal. 21 (1976), 316-329.

[18] I. Kluvánek and G. Knowles, Vector measures and control systems, Notas de Matemática, Vol. 58 (North-Holland, Amsterdam, 1975).

[19] D. R. Lewis, 'Integration with respect to vector measures', Pacific. J. Math. 33 (1970), 157-165.

[20] W. A. Luxemburg and A. C. Zaanen, Riesz spaces I (North-Holland, Amsterdam, 1971).

[21] P. Meyer-Nieberg, Banach lattices (Universitext, Springer, Berlin, Heidelberg, New York, 1991).

[22] I. Tweddle, 'Weak compactness in locally convex spaces', Glasgow Math. J. 9 (1968), 123-127.

[23] A. C. Zaanen, Riesz spaces II (North-Holland, Amsterdam, 1983).

Departamento Matemática Aplicada II

Escuela Superior de Ingenieros

Camino de los Descubrimientos, $\mathrm{s} / \mathrm{n}$

41092-Sevilla

Spain

e-mail: anfercar@matinc.us.es 